\title{
Herramientas de entrega, evaluación y calificación telemática
}

\author{
Pedro M. Cabezos Bernala ${ }^{a}$ Juan J. Cisneros-Vivób
}

a UPV•pcabezos@ega.upv.es buPV•jcisnero@ega.upv.es

\begin{abstract}
The aim of this article is to present a new software package, developed at the $U P V$, which let us to evaluate and to score the student's works in an efficient way, thus optimizing the time spent by the teacher to do these tasks. This software package also facilitates the student the on-line delivery of their works and speeds the reception by the teacher. This software package has tree modules or applications: the delivery module, the monitorization module and the evaluation and scoring module. The delivery module serves to send easily and properly the student's works. The monitorization module checks the delivery process allowing the teacher to download the works from the server, whereas the evaluation and scoring module provides a specific workbench to evaluate the works by means a customizable database to create type corrections and rubrics, which facilitates the evaluation and scoring process. After that, the student receives automatically a structured e-mail containing the evaluation and feedback provided by the teacher.
\end{abstract}

Keywords: evaluation, rubrics, scoring.

\section{Resumen}

La comunicación pretende dar a conocer un paquete de aplicaciones, desarrollado en la UPV, que permite evaluar y calificar trabajos de forma eficiente, optimizando el tiempo dedicado por el docente a estas tareas. Este paquete de aplicaciones también facilita a los alumnos la entrega telemática de los trabajos y agiliza su recepción por parte del profesor. Este paquete de aplicaciones informáticas consta de tres aplicaciones o módulos: el módulo de entrega, el módulo de monitorización y el módulo de evaluación y calificación. El módulo de entrega permite al alumno enviar sus trabajos, de manera rápida y sencilla. El módulo de monitorización controla el proceso de entrega y permite descargar los trabajos, mientras que el módulo de evaluación y calificación consiste en un entorno de trabajo que permite visualizar fácilmente los trabajos entregados y dispone de una base de datos para crear correcciones tipo y rúbricas personalizadas para agilizar la tarea de evaluación y calificación. El alumno recibe la calificación y evaluación 
Herramientas de entrega, evaluación y calificación telemática, basadas en rúbricas

del trabajo con el feedback proporcionado por el profesor mediante un email en el que se estructura toda la información.

Palabras clave: evaluación, rúbricas, calificación, telemática.

\section{Introducción}

Con el fin de agilizar y facilitar las tareas de entrega, evaluación y calificación de los trabajos realizados por los alumnos, se ha desarrollado un conjunto de aplicaciones informáticas que complementan y solventan algunas carencias detectadas en la plataforma de e-learning institucional Poliformat, basada en el entorno Sakai.

Este conjunto de aplicaciones está constituido por tres aplicaciones o módulos: El módulo de entrega, el módulo de monitorización y el módulo de evaluación y calificación.

El módulo de entrega permite al alumno enviar sus trabajos a un servidor, a disposición del profesor, de una manera sencilla y rápida. El alumno sólo tiene que autentificarse, arrastrar los archivos que desea enviar sobre la aplicación y pulsar un botón de envío.

El módulo de monitorización, controla el proceso de entrega y permite al docente acceder al servidor donde se han almacenado los trabajos enviados, para visualizarlos directamente desde el servidor o para realizar copias de los trabajos a un disco local. Esta aplicación permite también activar o desactivar la posibilidad de entrega en un momento dado.

Con el módulo de evaluación y entrega se define un entorno de trabajo que permite visualizar fácilmente los trabajos entregados y dispone de una base de datos para crear rúbricas y correcciones tipo personalizadas con las que agilizar la tarea de evaluación y calificación, además de permitir las aclaraciones puntuales. Haciendo uso de ello se genera un informe de evaluación de manera semi-automatizada con la evaluación y calificación del trabajo, que es almacenado y enviado automáticamente por email al alumno. Además de ello, la calificación del ejercicio se guarda automáticamente en una hoja de Excel.

\section{Objetivos}

- Facilitar al alumno la entrega de los trabajos vía telemática mediante un sencillo programa de entrega que no obligue al alumno a ir subiendo, uno a uno, cada archivo, como sucede actualmente con la plataforma Poliformat.

- Facilitar al profesor el acceso al servidor en el que se almacenan los trabajos y su copia masiva a un disco duro local, sin tener que recurrir al farragoso y lento proceso de descarga a través del servicio WebDAV, que resulta poco intuitivo y complicado para un gran número de profesores no familiarizados con estas herramientas de transmisión.

- Crear una aplicación específica para la evaluación y calificación de los trabajos con un entorno de trabajo personalizable que agilice el visionado de los trabajos integrando visores con el formato específico de los archivos y facilite las tareas de evaluación y 
calificación mediante el uso de rúbricas y la automatización de los procesos. En muchas ocasiones muchos alumnos suelen cometer los mismos fallos y el profesor debe realizar una misma puntualización repetidas veces cuando corrige. Ante esta situación, el profesor puede crear una corrección tipo, que podrá insertar automáticamente en el informe de evaluación que recibe el alumno.

- Facilitar al alumno la recepción de las evaluaciones y el feedback proporcionado por el profesor mediante un email que se genere y envíe automáticamente, de forma transparente, a medida que el profesor evalúa y califica los trabajos desde la aplicación.

- Almacenar las calificaciones de manera automatizada y transparente en un listado de Excel a medida que el profesor evalúa y califica los trabajos desde la aplicación.

\section{Desarrollo de la innovación}

La aplicación fue diseñada para satisfacer las necesidades del grupo de profesores que impartimos la asignatura Geometría Descriptiva en la ETSAV, tras haber analizado una serie de carencias y para intentar implementar una serie de ideas con el fin de automatizar y agilizar el proceso de evaluación.

En primer lugar fue necesario disponer de un servidor para almacenar los trabajos enviados por los alumnos; para ello se contó con la colaboración de los operadores informáticos de la Escuela Técnica Superior de Arquitectura, que pusieron a nuestra disposición una unidad de red en la que almacenar los archivos de forma segura. A esta unidad de red solamente tienen acceso total los profesores, mientras que los alumnos sólo tienen permiso de escritura a través del módulo de entrega, que realiza la copia de los archivos a este servidor.

La idea principal para el diseño del módulo de entrega fue que su uso resultara sumamente sencillo para el alumno y que permitiera subir múltiples archivos en un único proceso. Para ello se diseñó un interface en el que el alumno sólo debe introducir sus credenciales de la UPV, arrastrar los archivos que quiere enviar sobre la ventana del programa y pulsar el botón entregar (Fig.1).

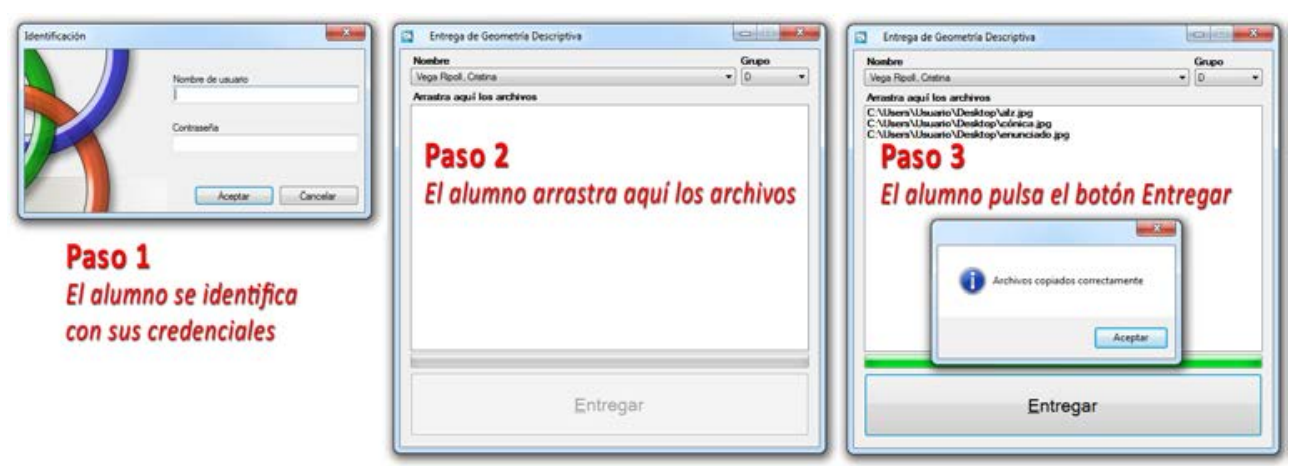

Fig. 1 Funcionamiento e interface del módulo de entregas que utilizan los alumnos

(cc) EY-NC-ND 2015, Universitat Politècnica de València

Congreso In-Red (2015) 
El programa crea automáticamente una carpeta en el servidor y copia allí los archivos proporcionados por el alumno, codificando el nombre de la carpeta con un formato concreto, que será utilizado posteriormente por los módulos de monitorización y de evaluación para gestionar los archivos. Esta codificación contiene, en primer lugar, el nombre del grupo al que pertenece el alumno, seguido del nombre. Todo este proceso es transparente para el alumno y una vez se ha efectuado la copia de los archivos el programa informa al alumno de que éstos han sido entregados correctamente.

El módulo de monitorización es de uso exclusivo para el profesor y le permite acceder al servidor para visualizar o realizar copias en local de los archivos entregados. El programa muestra, en tiempo real, el listado de las carpetas presentes en el servidor y el número de ficheros entregados por cada alumno, que se indica entre paréntesis tras el nombre del alumno (Fig.2).

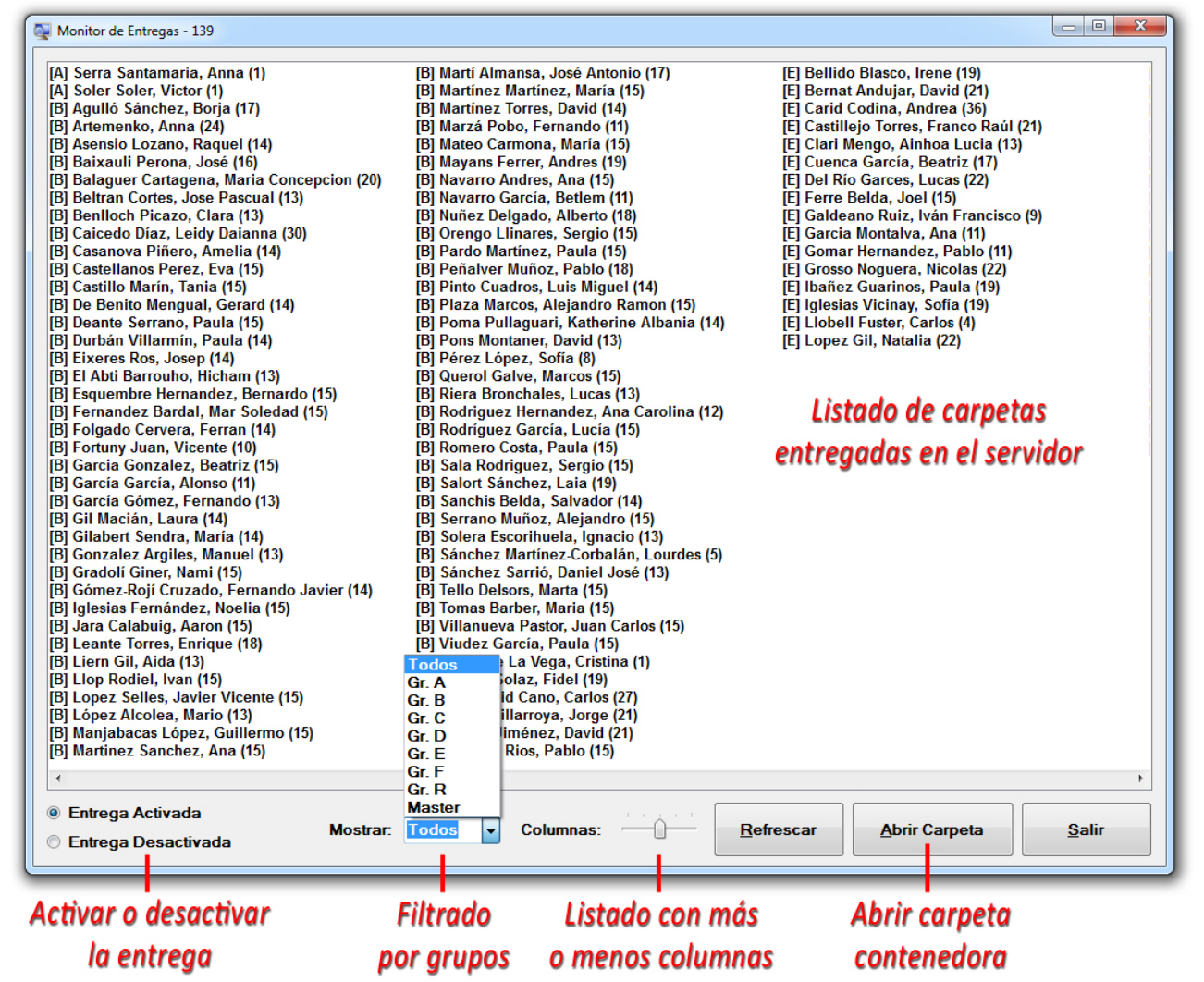

Fig. 2 Descripción del módulo de monitorización empleado por el profesor

Desde el módulo de monitorización se puede activar o desactivar la entrega, para que una vez que haya expirado el tiempo establecido para la entrega, el profesor pueda 
deshabilitarla. También se puede filtrar por grupos el listado y aumentar su número de columnas en caso de que haya un número muy elevado de carpetas.

Otra importante función del monitor de entregas es que permite abrir la carpeta remota contenedora de los trabajos como una unidad de red, de forma transparente, para que el profesor pueda descargar y acceder fácilmente a los archivos y carpetas generados, por lo que la copia de los archivos es mucho más sencilla, veloz y eficiente que el proceso a través de WebDAV ofrecido por Poliformat.

El diseño del interface del módulo de evaluación y calificación se realizó de modo que respondiera a las necesidades planteadas, como eran un acceso rápido a los trabajos, una visualización rápida y una elaboración del informe de evaluación semi-automático a través de la creación de una base de datos personalizada para almacenar correcciones tipo y el uso de rúbricas para la calificación de los trabajos.

El interface del módulo de evaluación y calificación tiene cuatro partes diferenciadas: el panel de exploración de archivos, el panel de vista previa, el panel de correcciones tipo y la ventana de texto para emitir el informe de evaluación (Fig.3).

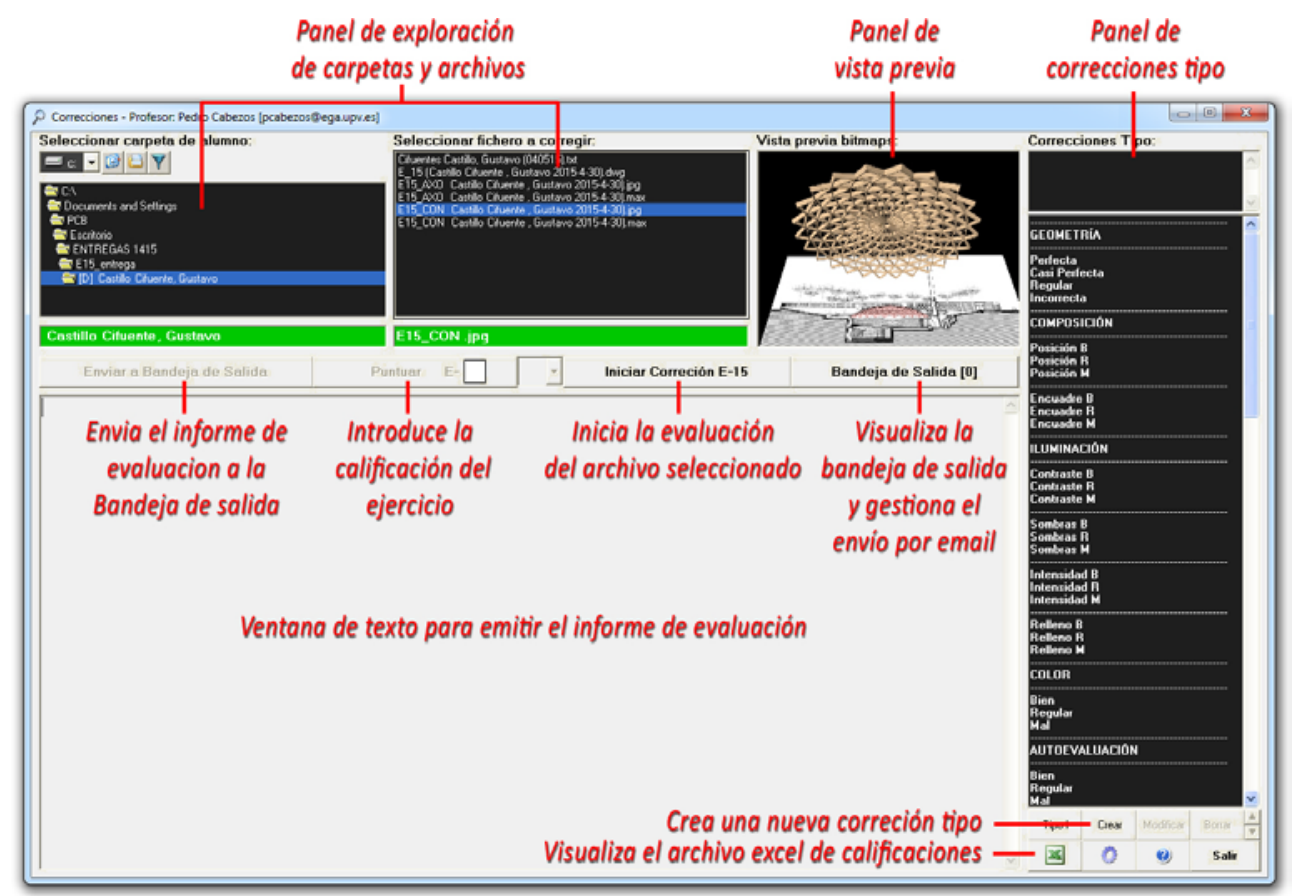

Fig. 3 Descripción del módulo de evaluación y calificación empleado por el profesor

En nuestro caso particular, la mayoría de los trabajos se almacenan en archivos gráficos ráster, como por ejemplo el formato JPG, por lo que se dotó al programa de un visor gráfico

(c)) EY-NC-ND 2015, Universitat Politècnica de València

Congreso In-Red (2015) 
integrado para visualizar de forma rápida y eficiente los archivos enviados por los alumnos. Este visor además de ofrecer una vista previa de la imagen, puede mostrarla también a pantalla completa, haciendo doble clic sobre el área de previsualización.

Además de las imágenes tipo ráster, se pueden evaluar también cualquier otro tipo de archivo, en nuestro caso suelen ser archivos gráficos vectoriales como el formato PDF de Adobe Acrobat, el archivo DWG de AutoCAD y el MAX de 3DStudio Max. Por ello para ver el contenido de este tipo de formatos, basta hacer doble clic sobre el archivo que se está evaluando, para que el programa abra el visor asociado al documento en cuestión.

Cuando el profesor utiliza el explorador de archivos integrado para situarse sobre la carpeta que contiene los archivos enviados por el alumno, el programa detecta automáticamente el nombre del alumno y grupo y se prepara para iniciar la evaluación del archivo que el profesor tenga seleccionado en ese momento.

En cuanto el profesor selecciona el archivo en cuestión y pulsa sobre el botón [Iniciar Corrección], el programa inserta automáticamente un encabezado en la ventana de texto que incluye el nombre del ejercicio que se está evaluando y a partir de este momento el profesor puede hacer uso del visor integrado para evaluar el trabajo y añadir sus indicaciones particulares sobre la ventana de texto, como en cualquier procesador de textos, pero resulta de mucha utilidad utilizar el panel lateral de correcciones tipo, que contiene una base de datos personal con las correcciones o indicaciones más frecuentes utilizadas por el profesor, que pueden insertarse de forma automatizada con sólo hacer doble clic en el título de la corrección tipo, situada dentro de este panel lateral.

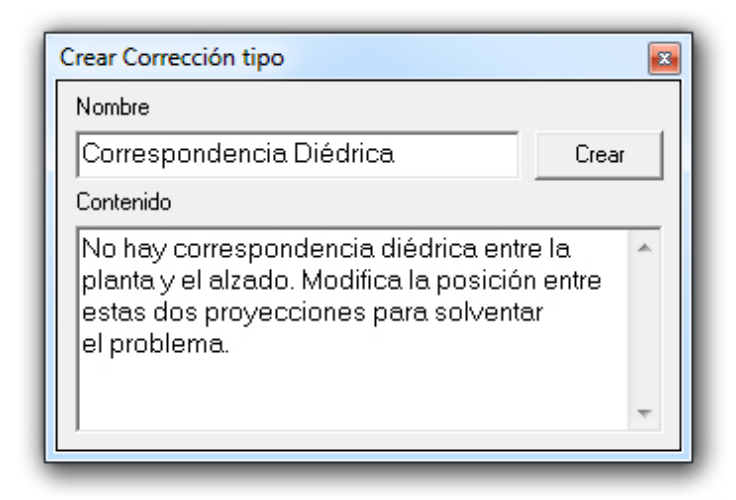

Fig. 4 Cuadro de dialogo para crear una corrección tipo

Para crear una corrección tipo, basta pulsar sobre el botón [Crear] y aparece un cuadro de dialogo (Fig.4), en el que se debe indicar el nombre o alias de la corrección tipo que aparecerá en el panel de correcciones, además del contenido del texto que será añadido automáticamente en el informe de evaluación cuando el profesor haga doble clic sobre su alias. Además de resultar cómodo para el profesor, esta herramienta nos sirve para 
identificar los errores más comunes cometidos por los alumnos, sobre los que deberemos incidir posteriormente.

El panel de correcciones puede contener evaluaciones en forma de rúbrica personalizada, de modo, que dependiendo del nivel de consecución alcanzado en cada apartado, se pueda establecer la calificación del ejercicio. En nuestro caso las rúbricas son consensuadas previamente con los alumnos, quienes, además de conocerlas, deben también aplicarlas, pues es una manera de desarrollar competencias transversales como el sentido crítico.

Una vez el profesor ha completado el informe de evaluación para el ejercicio, puede asignar la calificación mediante el botón [Puntuar], de modo que la calificación quedará incluida en el informe y ésta se almacenará automáticamente, de forma transparente, en un archivo Excel que contiene las calificaciones de todos los trabajos.

Por último el profesor envía el informe a la bandeja de salida mediante el botón [Enviar a bandeja de salida] y puede proseguir con la evaluación de otro trabajo. El profesor puede enviar en cualquier momento los informes generados por email de forma automatizada y transparente, pulsando el botón [Bandeja de Salida], con el que accederá a la lista de informes generados, y después [Enviar todos], con lo que cada informe se enviará automáticamente al alumno correspondiente. El programa guarda una copia del informe de evaluación en la carpeta que contiene el trabajo del alumno para el caso de revisiones posteriores.

\section{Resultados}

Una vez definidas las necesidades y completado el diseño de los interfaces, se programaron las aplicaciones utilizando el entorno de programación Microsoft Visual Studio .NET. Como resultado se obtuvieron las versiones compiladas de los tres módulos que hemos descrito y este conjunto de aplicaciones fue registrado en el Centro de Transferencia Tecnológica de la UPV mediante comunicación de resultado transferible.

Este conjunto de aplicaciones se viene utilizando y perfeccionado desde el año 2005 en la asignatura Geometría Descriptiva de la ETSA, para la entrega, evaluación y calificación de trabajos digitales gráficos, con una gran aceptación por parte de los profesores así como por parte de los alumnos. Prueba de ello son los resultados de las encuestas de satisfacción realizadas este mismo año a los alumnos y profesores que utilizan estas aplicaciones.

En primer lugar, se muestran los resultados de la encuesta realizada a los alumnos en relación con el módulo de entrega, que es el que ellos utilizan para entregar los trabajos de la asignatura.

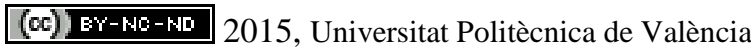

Congreso In-Red (2015) 
La entrega de los trabajos mediante el programa de entregas me ha resultado sencilla

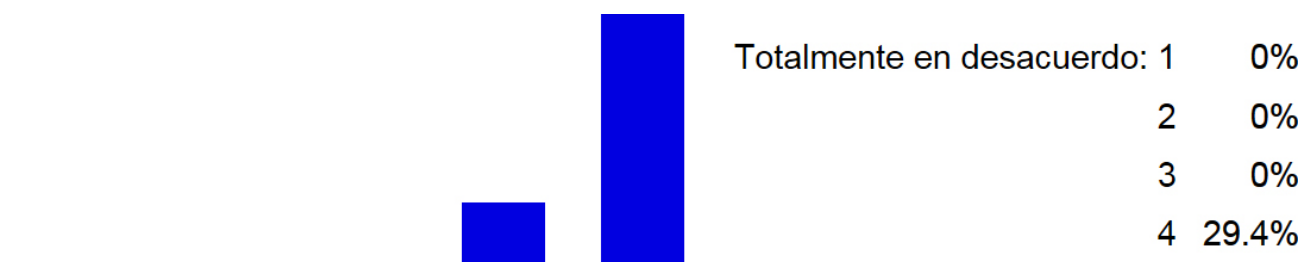

Totalmente de acuerdo: 5 70.6\%

\begin{tabular}{lllll}
\hline 1 & 2 & 3 & 4 & 5
\end{tabular}

El programa de entregas me ha permitido entregar los trabajos eficazmente

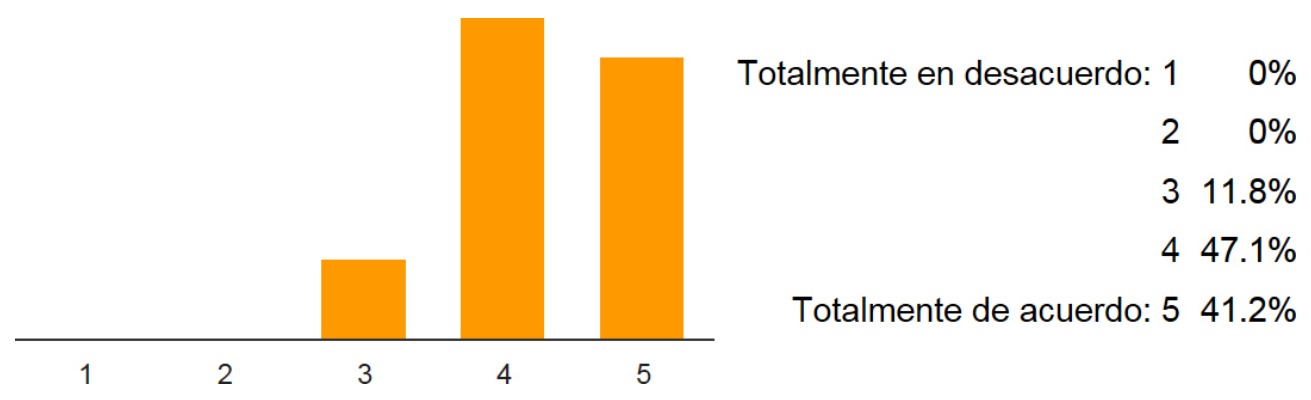

En comparación con la entrega de trabajos a través de Poliformat,

el funcionamiento del programa de entregas me resulta:

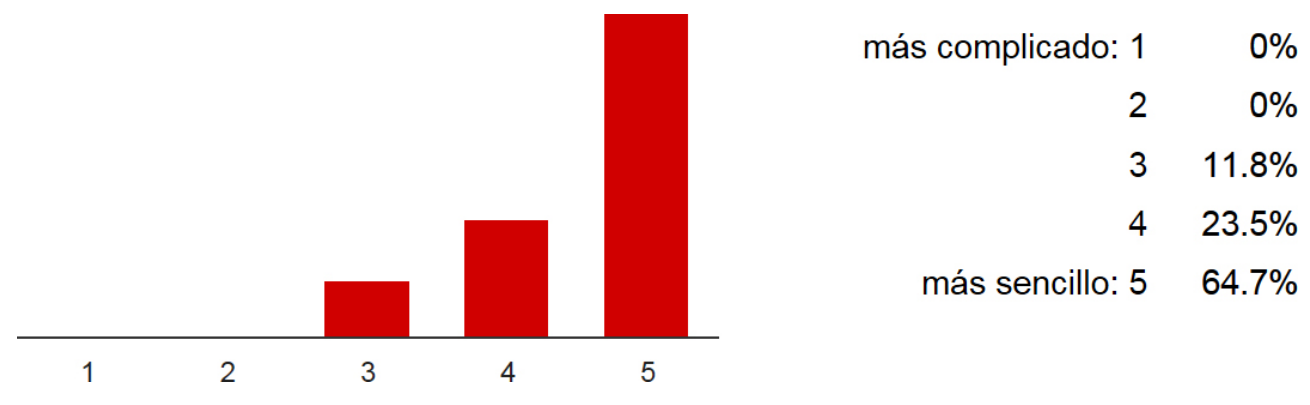

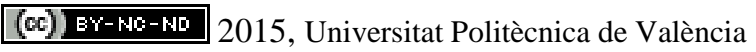


En comparación con la entrega de trabajos a través de Poliformat, el funcionamiento del programa de entregas me resulta:

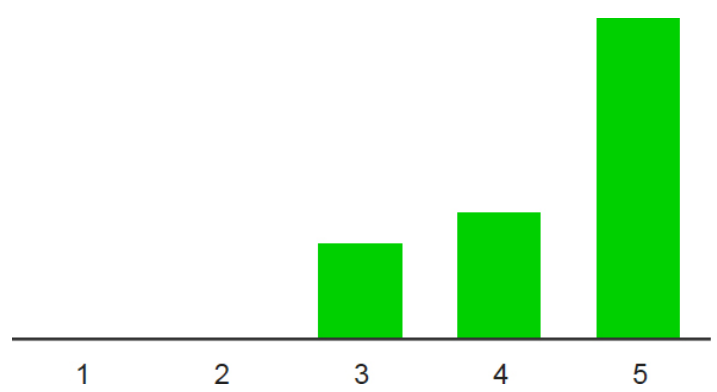
menos eficaz: $1 \quad 0 \%$
$20 \%$
$317.6 \%$
$423.5 \%$

más eficaz: $5 \quad 58.8 \%$

En comparación con la entrega de trabajos a través de Poliformat, el funcionamiento del programa de entregas me resulta:

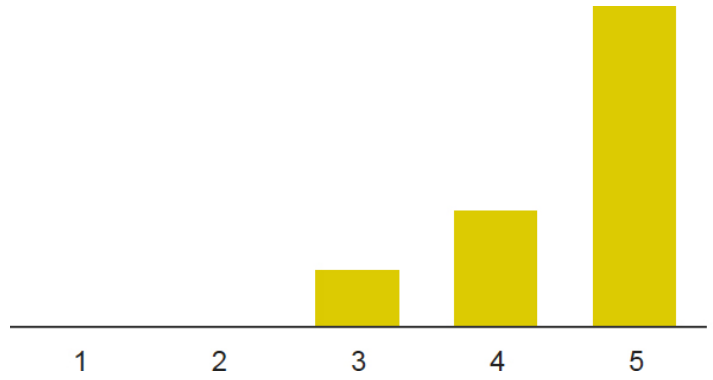
más lento: $1 \quad 0 \%$
$20 \%$
$311.8 \%$
$423.5 \%$

más rápido: $5 \quad 64.7 \%$

A continuación, se muestra la encuesta formulada a los profesores que utilizan estas herramientas acerca del funcionamiento del módulo de monitorización y el módulo de evaluación y calificación.

El módulo de monitorización me permite gestionar los archivos entregados por los alumnos con agilidad

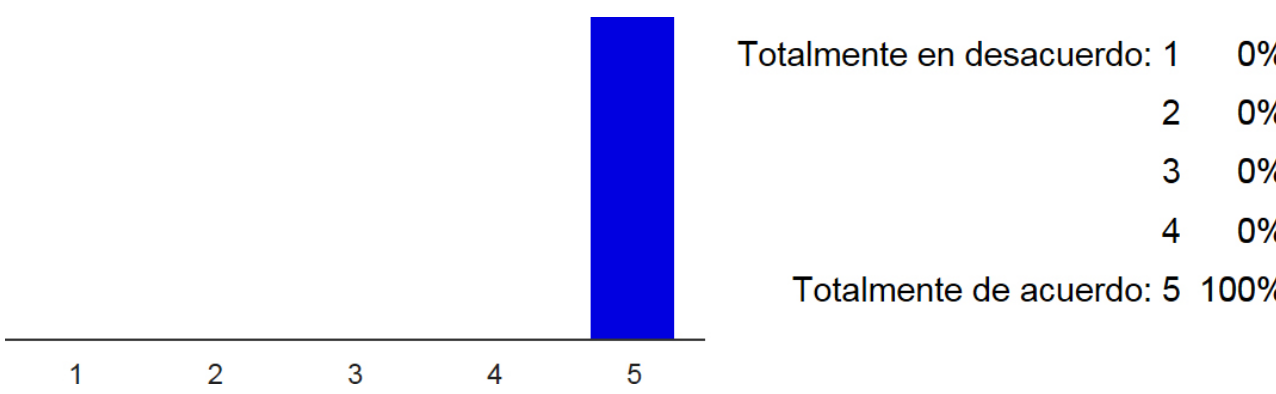

(c)) EY-NC-ND 2015, Universitat Politècnica de València

Congreso In-Red (2015) 
En comparación de la gestión de los archivos entregados por los alumnos a través de Poliformat, el uso del programa de entregas y monitorización me resulta:

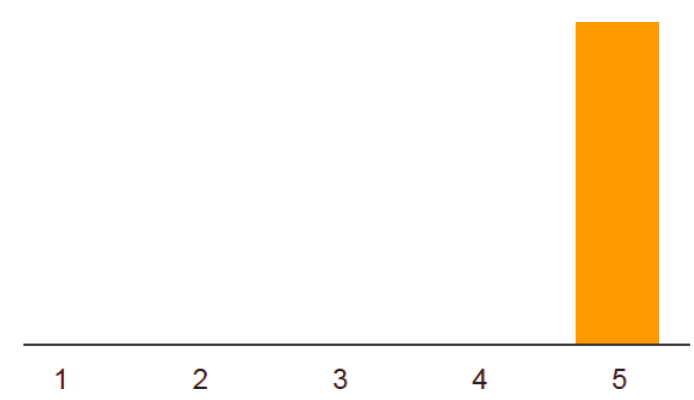

$\begin{array}{rr}\text { menos sencillo: } 1 & 0 \% \\ 2 & 0 \% \\ 3 & 0 \% \\ 4 & 0 \%\end{array}$

más sencillo: 5 100\%

En comparación de la gestión de los archivos entregados por los alumnos a través de Poliformat, el uso del programa de entregas y monitorización me resulta:

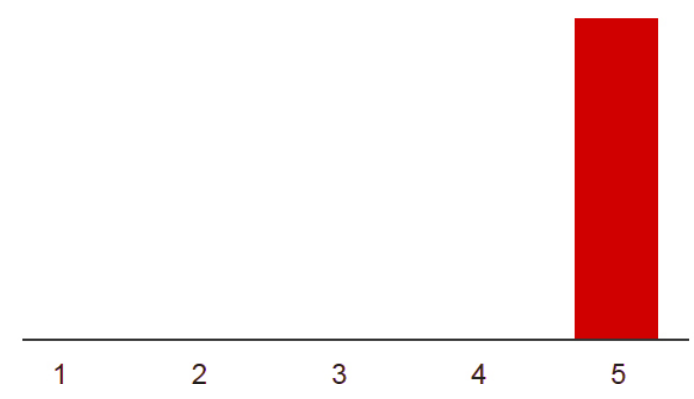

más lento: $1 \quad 0 \%$

$20 \%$

$30 \%$

$40 \%$

más rapido: 5 100\%

En comparación de la gestión de los archivos entregados por los alumnos a través de Poliformat, el uso del programa de entregas y monitorización me resulta:

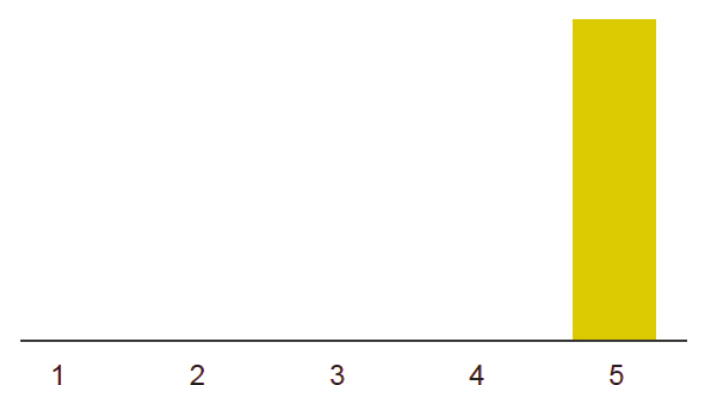

$\begin{array}{rr}\text { menos eficiente: } 1 & 0 \% \\ 2 & 0 \% \\ 3 & 0 \% \\ 4 & 0 \%\end{array}$

más eficiente: 5 100\% 
El funcionamiento y manejo del módulo de evaluación y calificación me resulta:

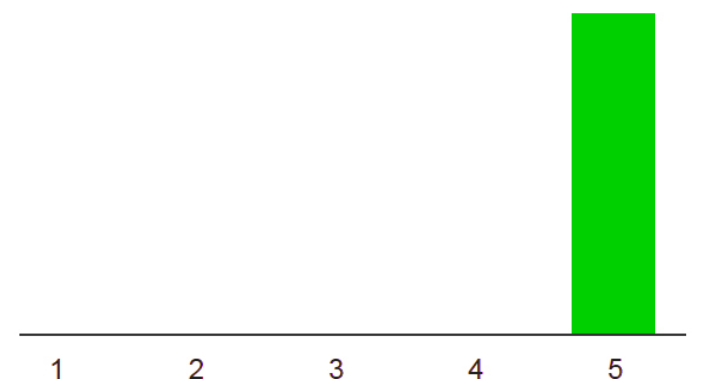

complejo: $1 \quad 0 \%$

$20 \%$

$30 \%$

$40 \%$

sencillo: 5 100\%

Considero que el módulo de evaluación y calificación agiliza la labor de evaluación y calificación de los trabajos

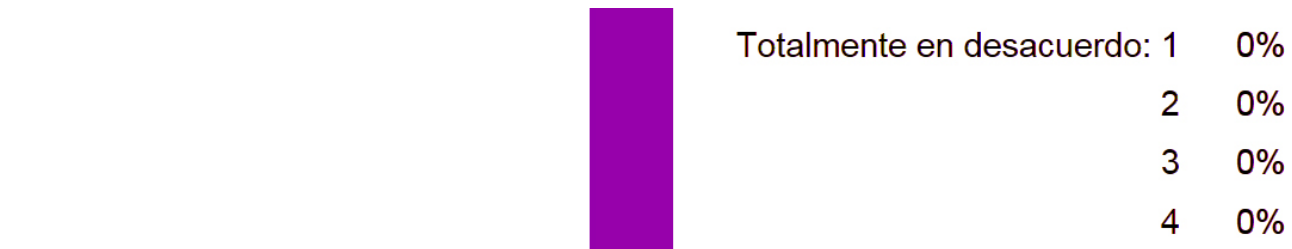

Totalmente de acuerdo: 5 100\%

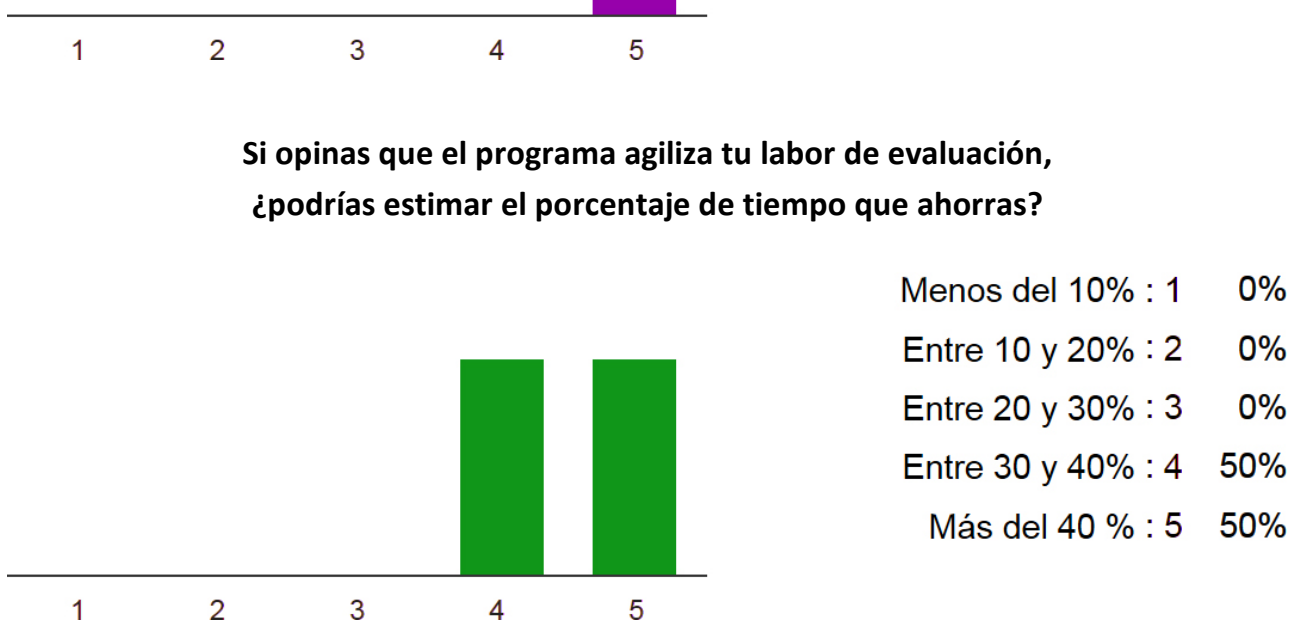

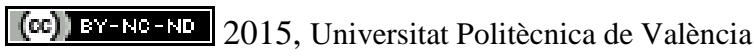

Congreso In-Red (2015) 
Herramientas de entrega, evaluación y calificación telemática, basadas en rúbricas

\section{Conclusiones}

A raíz de las encuestas realizadas se comprueba que, desde el punto de vista del alumno, se valora positivamente el módulo de entregas, tanto por la facilidad de uso como por la eficacia y rapidez en relación con la entrega desde la plataforma institucional Poliformat.

Desde el punto de vista del docente, la satisfacción es máxima ya que los procesos de gestión de archivos son más sencillos, rápidos y flexibles que los ofrecidos por Poliformat. Además de ello, el uso del módulo de evaluación y calificación agiliza notoriamente las tareas de corrección, produciendo un ahorro de tiempo muy considerable gracias a la automatización de los procesos, el uso de rubricas y la base de datos con correcciones tipo personalizadas.

Este conjunto de aplicaciones supone una verdadera innovación educativa, ya que el desarrollo de la aplicación es totalmente propio y no se basa en la utilización de ningún software desarrollado por terceros. 